\title{
A computed tomography-based spatial reference for pedicle screw placement in adolescent idiopathic scoliosis
}

\author{
Rob C. Brink ${ }^{1}$. Jelle F. Homans ${ }^{1}$ - Steven de Reuver ${ }^{1} \cdot$ Marijn van Stralen $^{2} \cdot$ Tom P. C. Schlösser $^{1} \cdot$ Max A. Viergever ${ }^{3}$. \\ Winnie C. W. Chu ${ }^{4}$ B Bobby K. W. $\mathrm{Ng}^{5} \cdot$ René M. Castelein ${ }^{1} \cdot$ Jack C. Y. Cheng ${ }^{5}$
}

Received: 9 February 2019 / Accepted: 14 July 2019 / Published online: 24 January 2020

(c) The Author(s) 2020

\begin{abstract}
Study design Cross-sectional.

Objectives To determine semiautomatically the 3D position of the pedicle axis in operative adolescent idiopathic scoliosis (AIS) patients relative to the operating table and the lamina, as orientation for pedicle screw placement for better understanding and reference of spine surgeons.

Summary of background data Pedicle morphology is well described as the angle between the convex and concave pedicle. However, the pedicle angle as relative to the neutral anterior-posterior axis or to an easy-to-use intravertebral landmark, remained unknown.

Methods The pedicles of the apex and two adjacent vertebrae cranial and caudal to the apex of 86 right-sided primary thoracic AIS curves were evaluated using semiautomatic 3D software on high-resolution CT scans, in the same prone position as during surgery. Pedicle vectors were obtained and calculated as transverse and sagittal angles, as relative to the neutral axis (corresponding with an axis perpendicular to the operating table) and to an axis perpendicular to the lamina.

Results At the apex, the mean convex and concave transverse pedicle angles were $14.3^{\circ}$ (95\% confidence interval [95\% CI]: 12.0-16.6) and $30.4^{\circ}(95 \%$ CI: $28.1-32.8)$ to the right. The angles decreased toward the adjacent levels cranial and caudal to the apex $(p<0.001)$ and linearly increased with a higher Cobb angle $(r \geq 0.472 ; p<0.001)$. The mean transverse pediclelamina angles, sagittal pedicle angles and the sagittal pedicle-lamina angles differed along the curve as well $(p<0.001)$.

Conclusions Pedicle angulation differs between convex and concave and depends on the position of the vertebra relative to the apex, as well as the curve severity. The transverse and sagittal pedicle angles, as relative to the operating table and laminae, could provide useful reference for better understanding of the distorted 3D morphology, and the angles, as given in this study, could serve as an approximate guideline for the expected direction of the pedicle screw.
\end{abstract}

Level of evidence Level IV.

Keywords Pedicle axis guideline $\cdot$ Lamina $\cdot$ Operating table $\cdot$ Computed tomography $\cdot$ Adolescent idiopathic scoliosis

Rob C. Brink

r.c.brink@umcutrecht.nl

1 Department of Orthopaedic Surgery, G05.228, University Medical Center Utrecht, P.O. Box 85500, 3508 GA Utrecht, The Netherlands

2 Imaging Division, University Medical Center Utrecht, Utrecht, The Netherlands

3 Image Sciences Institute, University Medical Center Utrecht, Utrecht, The Netherlands
4 Department of Imaging and Interventional Radiology, Prince of Wales Hospital, The Chinese University of Hong Kong, Shatin, Hong Kong

5 Department of Orthopaedics and Traumatology, Prince of Wales Hospital, The Chinese University of Hong Kong, Shatin, Hong Kong 


\section{Introduction}

Adolescent idiopathic scoliosis (AIS) is characterized by three-dimensional (3D) deformities in the vertebral column and trunk [1]. The abnormal vertebral body and pedicle morphology in AIS has been observed in cadaveric specimens for centuries and more recently from radiographic imaging studies [2-12]. Pedicle screw fixation at multiple levels has become one of the most widely used powerful techniques in spinal surgery for the 3D correction of AIS [13-18]. The complication rate due to pedicle screw misplacement in patients who underwent posterior instrumentation varies between 0 and $1 \%$; however, the rate of screw misplacement in the thoracic region varies between 5.7 and 50\% [18-25]. Complications related to the pedicle screw placement such as nerve root or spinal cord compression, pseudarthrosis, and major vessel injury are particularly more common in the more severe rigid curves. Studies aiming at more pathoanatomical 3D characterization of the pedicle length, pedicle angle, pedicle diameter, and ideal pedicle screw trajectories as well as the ideal pedicle screw entry points has been conducted [5, 7-10, 12, 26-33]. However, the pedicle angles in these previous studies are mostly described as the angle between the convex and concave pedicle, or as the angle between the pedicle and the vertebral axis. There is a lack of study of the 3D morphology and orientation of the pedicles with reference to the neutral axis of the human body and external neutral axis, like the clinically relevant prone position on the operating table. In 1987, Zindrick et al. [34] described the pedicle angles as relative to the neutral human body axis, but only for the nonscoliotic population. Knowledge of these pedicle angles and their 3D position with reference to the operating table as well as the lamina throughout the scoliotic curve could facilitate the intraoperative pedicle screw placement during posterior spinal surgery in AIS. It is important to notice that these data could provide useful reference for better understanding of the 3D morphology and orientation of the pedicles, for better understanding and reference of spine surgeons and not as the exact patient-specific screw trajectory. The objective of this study is to determine the 3D position of the pedicle axis in operative AIS patients relative to the operating table and the lamina, using semiautomatic 3D data generated from a large series of computed tomography (CT) scans of the whole spine of AIS patients taken in the prone position for preoperative navigation planning.

\section{Materials and methods}

\section{Study population}

The transverse and sagittal pedicle angles, as relative to the operating table and laminae, in this study could serve as an approximate guideline for pedicle screw placement in AIS. This study has been approved by the local research ethics committees. CT scans of AIS patients were selected from an existing database [35]. No extra imaging was made for the purpose of this study. All AIS patients who had received preoperative CT images (64 Slice Multi-detector CT scanner; GE Healthcare, Chalfont, St. Giles, United Kingdom; slice thickness $0.625 \mathrm{~mm}$ ) between June 2011 and March 2015, with primary thoracic curves (Lenke curve type 1-4) were included. The CT scans were acquired for navigationguided pedicle screw insertion in one of the participating centers. The scans were made in the same prone position as during surgery, to mimic the intraoperative position for posterior surgery and multilevel pedicle screw fixation. However, because of the limited space in the scanner, the intraoperative pads or pillows were not used. All patients had undergone routine upright posterior-anterior and bending radiography as well as supine magnetic resonance imaging of the full spine for detection of spinal cord abnormalities. Children with other spinal pathology, spinal trauma, previous spinal surgery, neurologic symptoms, neural axis abnormalities, syndromes associated with disorders of growth, or atypical left convex thoracic curves were excluded to obtain an as homogeneous population as possible. Therefore, the right pedicle is always the convex pedicle in this study and the left pedicle always the concave pedicle.

\section{CT measurement method}

The pedicle angles were measured semiautomatically (manual selection of the pedicle, automatic segmentation of the pedicle, and automatic calculation of the pedicle angle) for each individual pedicle by two trained observers, using inhouse developed software for semiautomatic analysis (ScoliosisAnalysis 5.1, Image Sciences Institute, Utrecht, the Netherlands), based on MeVisLab (MeVis Medical Solutions AG, Bremen, Germany). Four angles were measured of the convex and concave pedicles; the transverse and sagittal pedicle angle as relative to the neutral axis, corresponding with an axis perpendicular to the operating table, and the transverse and sagittal angle between the pedicle axis and the lamina (Figs. 1, 2, 3). This process consisted of six steps. First, the observer selected the pedicles of the apex and the pedicles of the adjacent two vertebrae cranial and caudal to the apex on the CT scan, using software developed for this purpose. Second, the observer manually selected the approximate location of the longitudinal line through each pedicle. This axis was not used as pedicle axis, but served to initialize the computerized method by defining the location of the pedicle in the 3D image. Third, the software automatically generated a 3D reproduction of that pedicle, dilated an imaginary cylinder inside the pedicle until it reaches the outer cortex of the pedicle, and calculated the vector of the 
Fig. 1 Pedicle angles were measured using the in-house software developed for this study. The observer manually selected the approximate location of the longitudinal axis through each pedicle. This axis was not used as pedicle axis but served to initialize the computerized method by defining the location of the pedicle in the 3D image. Next, the software automatically generated a 3D reproduction of the pedicle, blew up an imaginary cylinder inside the pedicle until it hit the outer cortex of the pedicle, and calculated the vector of the axis straight through the imaginary cylinder, obtained in an $3 \mathrm{D}$ coordinate system. Positive transverse angles indicate angulation to the concave (left) side of the patient, and negative values indicate angulation to the convex (right) side. Positive sagittal angles indicate angulation to the cranial side, and negative values indicate angulation to the caudal side
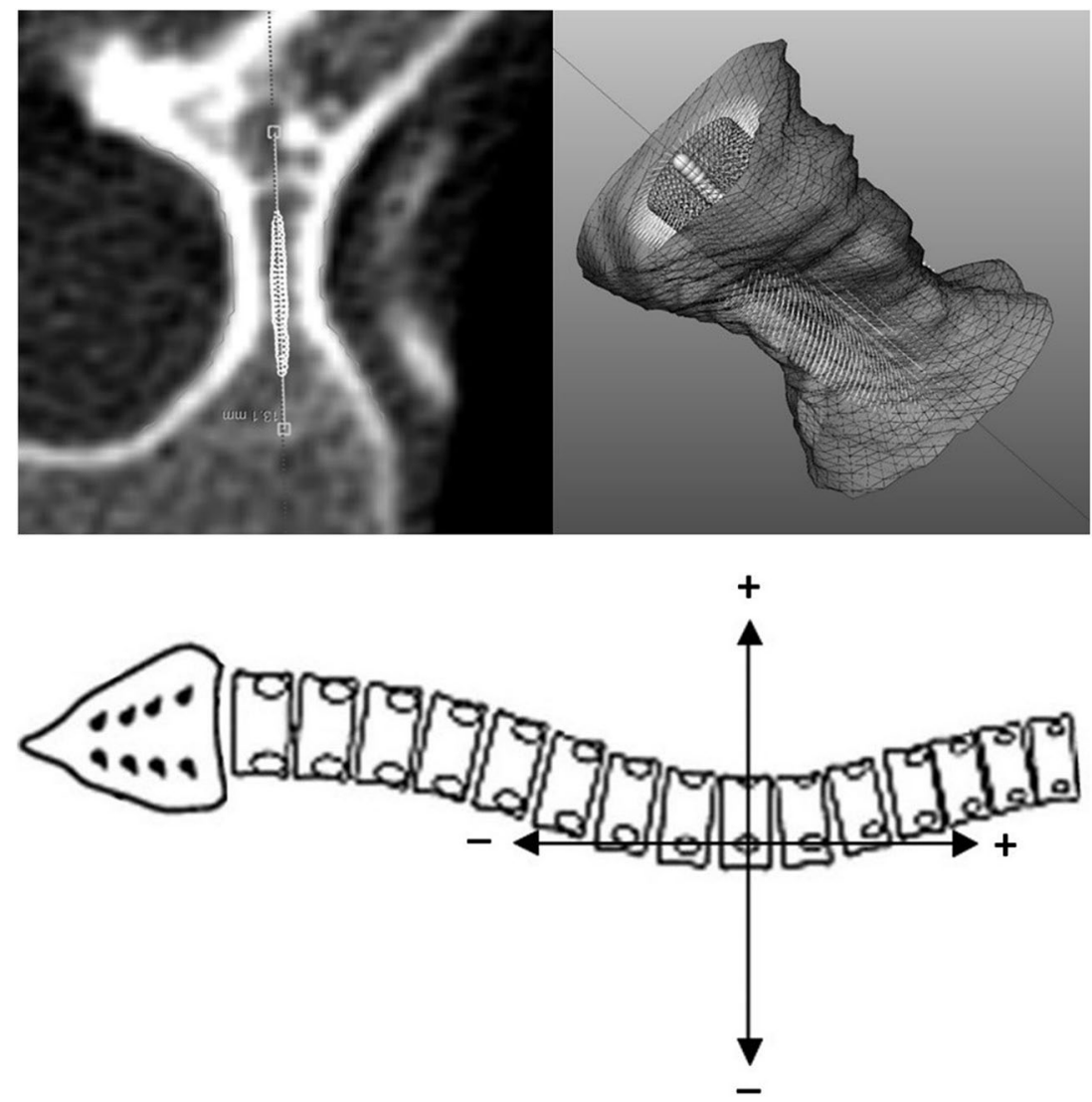

axis through the imaginary cylinder, obtained in a 3D coordinate system (Fig. 1). Fourth, the transverse pedicle angle was measured, as the angle between the transverse pedicle axis and the anterior-posterior axis of the sacral plate, which is considered as neutral in the scoliotic spine, and is perpendicular to the operating table (Fig. 2) [36, 37]. Fifth, the sagittal pedicle angle was measured, as the angle between the sagittal pedicle axis and a line perpendicular to the scanning table, corresponding with the operating table, because the prone CT scanning position corresponds with the prone intraoperative position (Fig. 3). Last, as an intravertebral reference, the pedicle-lamina angles were calculated. In the transverse plane, the angle between the pedicle axis and a line perpendicular to the lamina axis was measured. The lamina axis was defined as the line connecting the most ventral part of the posterior laminar cortex of each lamina at the junction to the transverse processes. In the sagittal plane, it was defined as the angle between the pedicle axis and a line perpendicular to the lamina of the corresponding level and one level to cranial (Figs. 2, 3). Positive transverse angles indicate angulation to the concave (left) side of the patient, negative values indicate angulation to the convex (right) side. Positive sagittal angles indicate angulation to the cranial side, and negative values indicate angulation to the caudal side. For intra- and interobserver reliability, the observers analyzed a random subset of $10 \mathrm{CT}$ scans on separate sittings (observer 1 twice and observer 2 once).

\section{Statistical analysis}

The statistical analyses in this study were done in SPSS 23.0 for Windows (SPSS Inc., Chicago, IL). Pedicle angle differences between different levels at the apex and the adjacent cranial and caudal levels, as well as the differences between convex and concave, were analyzed with a one-way repeated measured analysis of variance (ANOVA) added with a follow-up pairwise comparison and post hoc Bonferroni correction between each level. ANOVA, including as post hoc Bonferroni correction, was used as well for analyzing the pedicle angle differences between different Cobb angle subgroups and different curves, based on the level of the apex and length of the curve. Pearson correlation coefficient $(r)$ defined the relationship between the different pedicle angles and the Cobb angle. Finally, intra- and interobserver variabilities were obtained as intraclass correlation coefficients. The statistical significance level was set at 0.05 . 
Fig. 2 Two transverse angles are measured: the transverse pedicle angle and the transverse pedicle-lamina angle. The transverse pedicle angle was measured as the angle between the transverse pedicle axis and the anterior-posterior axis of the sacral plate, which is considered as neutral in the scoliotic spine and is perpendicular to the operating table (I). The pedicle-lamina angles were calculated in the transverse plane as the angle between the pedicle axis and a line perpendicular to the lamina axis (II). Positive transverse angles indicate angulation to the concave (left) side of the patient, and negative values indicate angulation to the convex (right) side

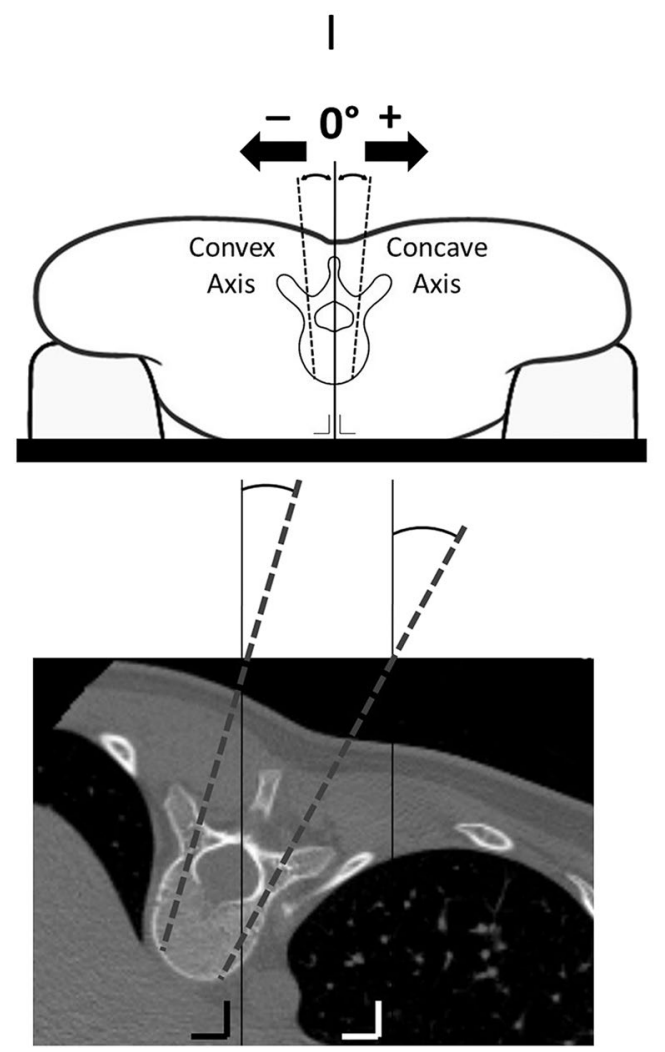

\section{Results}

\section{Study population}

The database consisted of CT scans of 86 AIS patients with primary right thoracic (Lenke type 1-4) curves (Table 1). The level of apex varied between T7 and T11 and the length of the curves varied between 5 and 10 vertebrae.

\section{Transverse pedicle angle relative to the neutral operating table}

At the apex, the mean angle between the transverse pedicle axis and the neutral axis was $14.3^{\circ}$ (95\% confidence interval $\left.[\mathrm{CI}] 12.0^{\circ}, 16.6^{\circ}\right)$ on the convex side and $30.4^{\circ}(95 \%$ CI $\left.28.1^{\circ}, 32.8^{\circ}\right)$ on the concave side and was significantly larger compared with the two levels cranial and caudal to the apex $(p<0.001$; Table 2$)$. The Cobb angle had a positive correlation with the pedicle angle at the apex (convex pedicle: $r=0.472, p<0.001$; and concave pedicle: $r=0.508$, $p<0.001)$. The rest of the levels showed a weak or nonsignificant correlation with the Cobb angle (the angles based on different curve severity groups are shown in Fig. 4). The level of apex varied between $\mathrm{T} 7$ and $\mathrm{T} 11$ and the curve length between 5 and 10 vertebrae. However, the apical transverse pedicle axis did not differ between the curves with different apical levels ( $p \geq 0.120$ ), or between curves with different lengths $(p \geq 0.897)$.

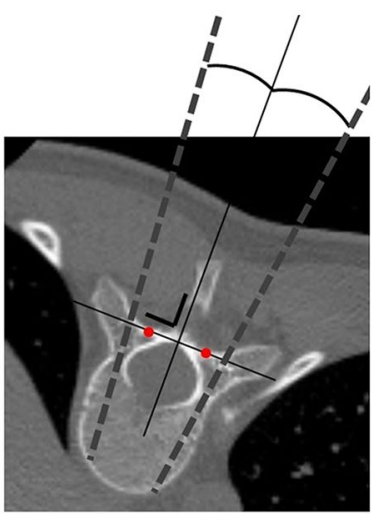

\section{Sagittal pedicle angle relative to the neutral operating table}

At the apex, the mean angle between the sagittal pedicle axis and the neutral plane was $-6.7^{\circ}\left(95 \% \mathrm{CI}-8.3^{\circ},-5.0^{\circ}\right)$ on the convex side and $-6.2^{\circ}\left(95 \% \mathrm{CI}-8.1^{\circ},-4.4^{\circ}\right)$ on the concave side and was significantly larger as compared with the two levels cranial and caudal to the apex $(p \leq 0.027)$, except for the convex pedicle one level cranial to the apex $(p=0.492)$ and the concave pedicle two levels cranial to the apex $(p=0.312$; Table 3$)$. Weak or nonsignificant correlations were observed between the sagittal pedicle angle and the Cobb angle (the angles based on different curve severity groups are shown in Fig. 5). The level of the apex and the length of the curves did not significantly influence the sagittal pedicle axis $(p \geq 0.220)$.

\section{Pedicle-lamina angles}

The transverse pedicle-lamina angle was on average larger at the apex as compared with one and two levels cranial and caudal to the apex $(p<0.001)$, except the convex pedicle one 
Fig. 3 Two sagittal angles are measured: the sagittal pedicle angle and the sagittal pedicle-lamina angle. The sagittal pedicle angles were measured as the angle between the sagittal pedicle axis and the line perpendicular to the scanning table, corresponding with the operating table, because the prone CT scanning position corresponds with the prone intraoperative position (I). The sagittal pedicle-lamina angles were calculated in the sagittal plane as the angle between the pedicle axis and a line perpendicular to the lamina axis between the lamina of that level and one level beneath (II). Positive sagittal angles indicate angulation to the cranial side, and negative values indicate angulation to the caudal side
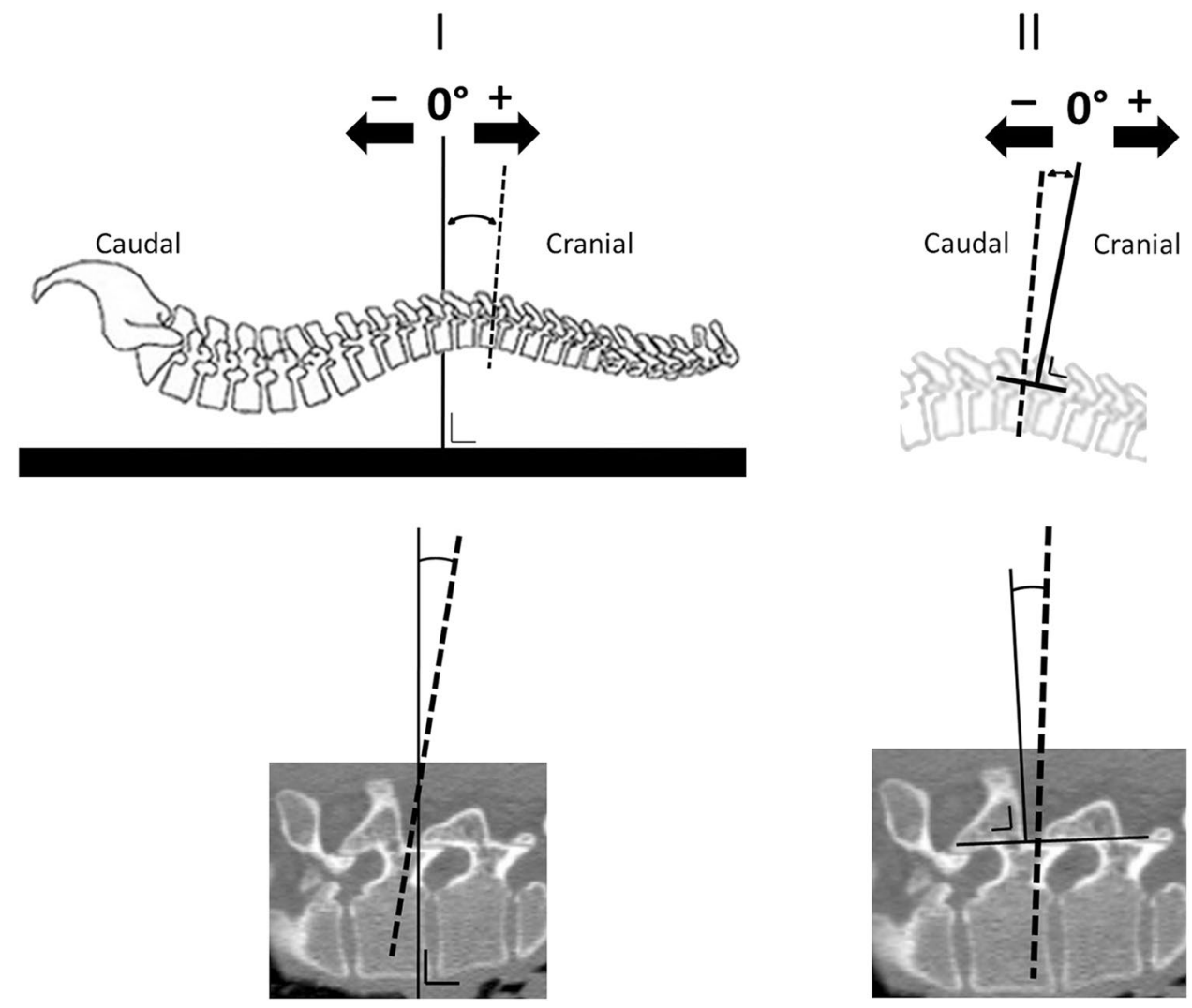

and two levels caudal to the apex ( $p=0.089$ and $p=0.200$; Table 2). The sagittal pedicle-lamina angle was on average larger at the apex, as compared with the adjacent cranial and caudal vertebrae $(p<0.001$; Table 3$)$. Only weak or nonsignificant correlations were observed between the transverse pedicle-lamina angle and the Cobb angle (the angles based on different curve severity groups are shown in Figs. 4 and $5)$. The level of the apex and the length of the curves did not influence the sagittal pedicle axis $(p \geq 0.181)$.

\section{Reliability}

Intraclass correlation coefficients for intra- and interobserver reliabilities were 0.93 (95\% confidence interval $0.90,0.95$ ) and $0.94(0.91,0.96)$ for the pedicle axes as relative to the operating table, and $0.97(0.95,0.98)$ and $0.95(0.93,0.97)$ for the pedicle axes as relative to the lamina.

\section{Discussion}

Several studies already provide accurate descriptions of pedicle morphology in AIS as the angle between the convex and concave pedicle or as the angle between the pedicle and the vertebral body axis [5, 7-10, 12, 26-29]. However, pedicle orientation throughout the curve in AIS, as compared with the neutral axis or operating table, like Zindrick et al. [34] did for the nonscoliotic spine in 1987, remained undetermined so far in AIS. Accurate description of pedicle orientation and morphology is important for pedicle screw placement during the surgical treatment of AIS. Therefore, the 3D orientation of pedicles in moderate to severe primary thoracic AIS curves, as compared with the neutral axis (perpendicular to the operating table), as well as the lamina, using high-resolution CT scans in the same prone position as during the surgery was reported in this study.

The mean apical transverse pedicle angle was $14.3^{\circ}(95 \%$ CI $\left.12.0^{\circ}, 16.6^{\circ}\right)$ on the convex side and $30.4^{\circ}\left(95 \%\right.$ CI $28.1^{\circ}$, $32.8^{\circ}$ ) on the concave side and decreased toward the junction zone. This angulation increased in curves with a higher Cobb angle. The sagittal pedicle angle as well as the angles between the pedicle and the lamina differed along the curve as well. However, the level of the apex and the length of the curve did not influence the pedicle angles of the apex. The data support that it is not the anatomical vertebral level on its own (T7, T8, etc.) but the vertebral level in relation with the apex (apex, or one or two levels cranial or caudal to the apex) that influences the pedicle axis. Although this study is the first in describing the pedicle angle as compared with the neutral body axis or operating table, the angle differences between the convex and concave pedicle $\left(12^{\circ}-17^{\circ}\right)$ were in 
Table 1 Demographics and curve characteristics are shown for all included adolescent idiopathic scoliotic (AIS) patients

\begin{tabular}{ll}
\hline & AIS patients $(n=86)$ \\
\hline Age, years & \\
Range & $10-26$ years \\
Mean \pm SD & $16.2 \pm 2.9$ years \\
Females & $73(85 \%)$ \\
Thoracic curve right convexity & $86(100 \%)$ \\
Primary thoracic Cobb angle & $46^{\circ}-109^{\circ}$ \\
Range & $46^{\circ}-109^{\circ} 6$ \\
& $9.4 \pm 12.3^{\circ}$ \\
Mean \pm SD & $69.4^{\circ} \pm 12.3^{\circ}$ \\
Cobb angle group & \\
$<60^{\circ}$ & $15(17 \%)$ \\
$60^{\circ}-70^{\circ}$ & $39(46 \%)$ \\
$70^{\circ}-80^{\circ}$ & $15(17 \%)$ \\
$>80^{\circ}$ & $17(20)$ \\
Lenke curve & \\
Type 1 & $43(50 \%)$ \\
Type 2 & $26(30 \%)$ \\
Type 3 & $12(14 \%)$ \\
Type 4 & $5(6 \%)$ \\
Level of the apex & \\
Thoracic 7 & $7(8 \%)$ \\
Thoracic 8 & $23(27 \%)$ \\
Thoracic 9 & $34(40 \%)$ \\
Thoracic 10 & $21(24 \%)$ \\
Thoracic 11 & $1(1 \%)$ \\
\hline &
\end{tabular}

$S D$ standard deviation

Unless otherwise noted, values are $n(\%)$

general similar to the findings as described in previous studies [7, 27, 38]. Takeshita et al. [9] already measured the direction of the thoracic and lumbar pedicles as the angle between the pedicle axis and a line connecting both sides of the middle of the superior facet base, using CT imaging, independent of the location of the apex of the curve. Because the direction of the pedicles is related to the rotation of the vertebra, and, therefore, related to the position of the vertebrae within the curve, the current study measured the pedicle direction of the apex and the two levels cranial and caudal to the apex. Using in-house developed software for semiautomatic analysis to construct 3D pedicle images and calculate the vector, the measurements are accurate and less vulnerable to observer subjectivity, compared with the use of 2D transverse images and measuring pedicle angles by hand in AIS. We believe the manual method has greater potential for measurement error. Previous studies have shown that the alignment of the scoliosis is influenced by the position of the patient [39-43]. In this study, the CT scans were made in the same prone position as the position during surgery.

Previous studies described the pedicle morphology as the angulation between the convex and concave pedicles or as the angulation between the pedicle and the vertebral body axis [7, 27, 38]. In the current study, the pedicle angulation was measured as relative to the sacral axis, in the transverse plane. Because the sacrum is considered as neutral in the scoliotic spine and the position of the patient during scanning and surgery are in the same prone positions, the sacral axis is perpendicular to the operating table [36, 37]. Therefore, the data of the current study could be used for better understanding of the distorted 3D pedicle morphology for the spine surgeon. Several methods were described to insert pedicle screws. Di Silvestre et al. [20] described a mini-laminotomy, using a spatula to palpate the borders of the thoracic pedicles. The data of this current study could not be used for the exact pedicle trajectory, but provide useful reference for better understanding of the pedicle morphology and orientation, as relative to the surgery table as well as the lamina. These data facilitate a spatial reference for the intraoperative pedicle screw placement during posterior spinal surgery in AIS. From there, the surgeon has to follow the screw trajectory of the specific patients, using several techniques, like the spatula method as described by
Table 2 Mean transverse pedicle angles, as relative to the neutral anterior-posterior axis, as well as the pedicle-lamina angles, including $95 \%$ confidence intervals, of the convex and concave pedicles in the primary thoracic curves are shown for the apex and the two adjacent cranial $(+1$ and +2$)$ and caudal $(-2$ and -1$)$ levels

\begin{tabular}{|c|c|c|c|c|c|c|}
\hline & \multicolumn{3}{|c|}{ Transverse pedicle angle $\left(^{\circ}\right)$} & \multicolumn{3}{|c|}{ Transverse pedicle-lamina angle $\left(^{\circ}\right)$} \\
\hline & Convex & Concave & $p$ & Convex & Concave & $p$ \\
\hline+2 & $1.3(-0.6,3.2)$ & $18.2(16.3,20.1)$ & $<0.001$ & $-8.4(-10.2,-6.6)$ & $8.5(6.9,10.1)$ & $<0.001$ \\
\hline+1 & $8.0(6.1-, 10.0)$ & $25.2(23.2,27.2)$ & $<0.001$ & $-7.7(-9.1,-6.3)$ & $9.5(8.0,11.0)$ & $<0.001$ \\
\hline Apex & $14.3(12.0,16.6)$ & $30.4(28.1,32.8)$ & $<0.001$ & $-3.9(-5.0,-2.8)$ & $12.2(10.8,13.6)$ & $<0.001$ \\
\hline-1 & $10.0(7.9,12.1)$ & $24.5(22.3,26.7)$ & $<0.001$ & $-5.4(-6.6,-4.1)$ & $9.1(7.6,10.7)$ & $<0.001$ \\
\hline-2 & $4.5(2.2,6.7)$ & $16.6(14.4,18.8)$ & $<0.001$ & $-5.1(-6.7,-3.6)$ & $7.0(5.5,8.6)$ & $<0.001$ \\
\hline
\end{tabular}

Positive transverse angles indicate angulation to the concave (left) side of the patient, and negative values indicate angulation to the convex (right) side. The $p$ values were calculated for the differences between the convex and concave pedicle 
Fig. 4 Mean convex and concave transverse pedicle angles and transverse pedicle-lamina angles of the apex and the two cranial $(+1$ and +2$)$ and caudal $(-1$ and -2$)$ levels are shown for different curve severities (Cobb angle $<60^{\circ}$, Cobb angle $60^{\circ}-70^{\circ}$, Cobb angle $70^{\circ}-80^{\circ}$, and Cobb angle $>80^{\circ}$ )

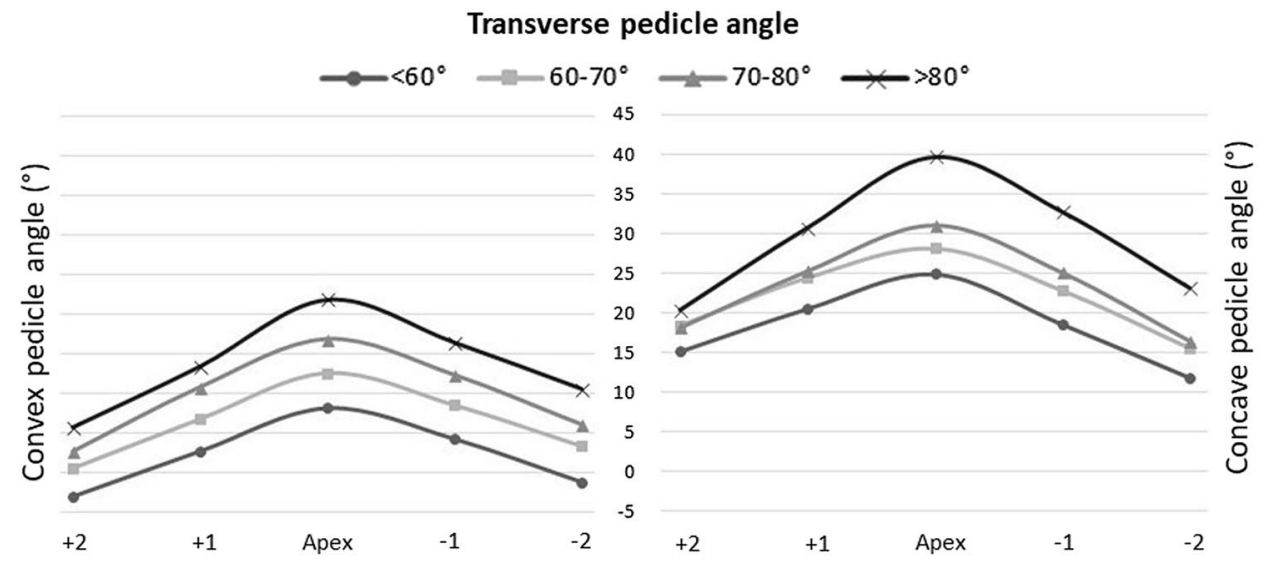

Transverse pedicle-lamina angle

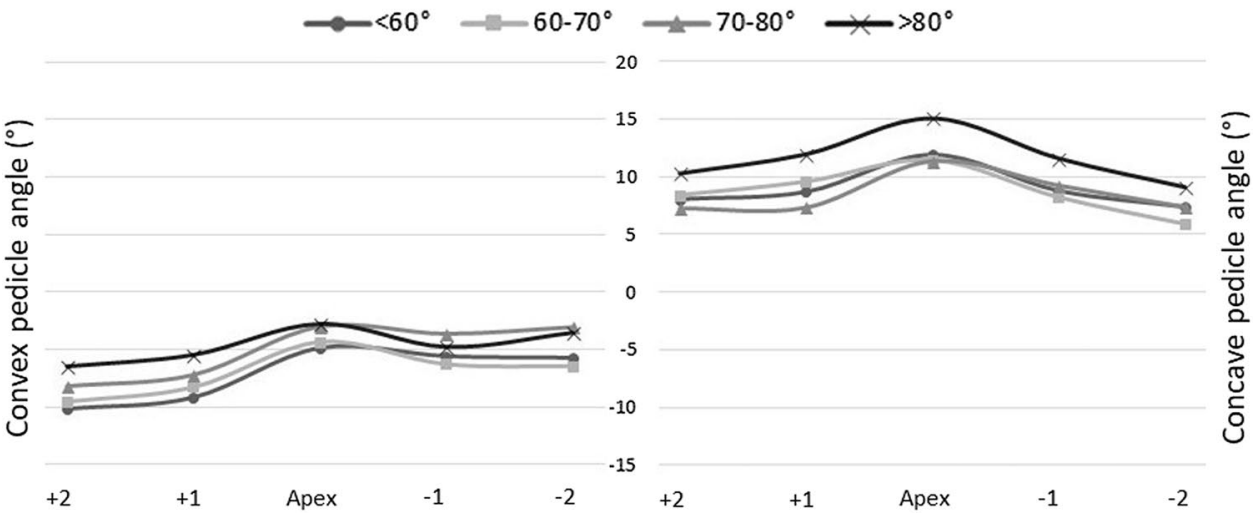

Di Silvestre et al. The reference data of this study may help to support the free-hand technique and to reduce the intraoperative radiation. Additionally, the pedicle-lamina angle, as derived from this study, could be used for in vivo pedicle screw positioning during surgery as well. Because the pedicle angle differed within the curve, the pedicle angles were shown for different groups of patients, based on the Cobb angle. For this study, an already existing CT database was used, acquired as part of the general preoperative workup for

Table 3 Mean sagittal pedicle angles, as relative to the neutral anterior-posterior axis, as well as the pedicle-lamina angles, including 95\% confidence interval, of the convex and concave pedicles in the navigation-guided surgery in one of our institutions, resulting in a cross-sectional study design. No additional imaging was made for the purpose of this study. If a pedicle is too narrow for the pedicle screw, or the pedicle or lamina are too much deformed, the surgeon could decide to select an extra-pedicle trajectory between the rib head and the pedicle, parallel to the end plate of the vertebra in the sagittal plane and directed toward the midline in the transverse plane, to maximize the length as well as bone purchase. A limitation

primary thoracic curves are shown for the apex and the two adjacent cranial $(+1$ and +2$)$ and caudal $(-2$ and -1$)$ levels

\begin{tabular}{|c|c|c|c|c|c|c|}
\hline & \multicolumn{3}{|c|}{ Sagittal pedicle angle $\left({ }^{\circ}\right)$} & \multicolumn{3}{|c|}{ Sagittal pedicle-lamina angle $\left(^{\circ}\right)$} \\
\hline & Convex & Concave & $p$ & Convex & Concave & $p$ \\
\hline+2 & $-2.2(-3.9,-0.6)$ & $-8.4(-10.2,-6.7)$ & $<0.001$ & $-20.3(-22.4,-18.2)$ & $-27.6(-30.0,-25.1)$ & $<0.001$ \\
\hline+1 & $-5.5(-7.1,-3.9)$ & $-9.8(-11.6,-8.1)$ & $<0.001$ & $-13.4(-15.4,-11.4)$ & $-18.6(-21.1,-16.2)$ & $<0.001$ \\
\hline Apex & $-6.7(-8.3,-5.0)$ & $-6.2(-8.1,-4.4)$ & 0.437 & $-4.3(-6.5,-2.1)$ & $-1.9(-4.8,0.90)$ & 0.007 \\
\hline-1 & $-3.5(-5.3,-1.7)$ & $-1.4(-3.0,0.3)$ & $<0.001$ & $8.6(6.3,11.1)$ & $14.2(11.9,16.6)$ & $<0.001$ \\
\hline-2 & $-4.0(-5.9,-2.2)$ & $1.3(-0.5,3.0)$ & $<0.001$ & $17.1(14.8,19.4)$ & $20.1(17.5,22.8)$ & 0.01 \\
\hline
\end{tabular}

Positive sagittal angles indicate angulation to the cranial side, and negative values indicate angulation to the caudal site. The $p$ values were calculated for the differences between convex and concave pedicle 
Fig. 5 Mean convex and concave sagittal pedicle angles and transverse pedicle-lamina angles of the apex and the two cranial $(+1$ and +2$)$ and caudal $(-1$ and -2$)$ levels are shown for different curve severities (Cobb angle $<60^{\circ}$, Cobb angle $60^{\circ}-70^{\circ}$, Cobb angle $70^{\circ}-80^{\circ}$, and Cobb angle $>80^{\circ}$ )
Sagittal pedicle angle

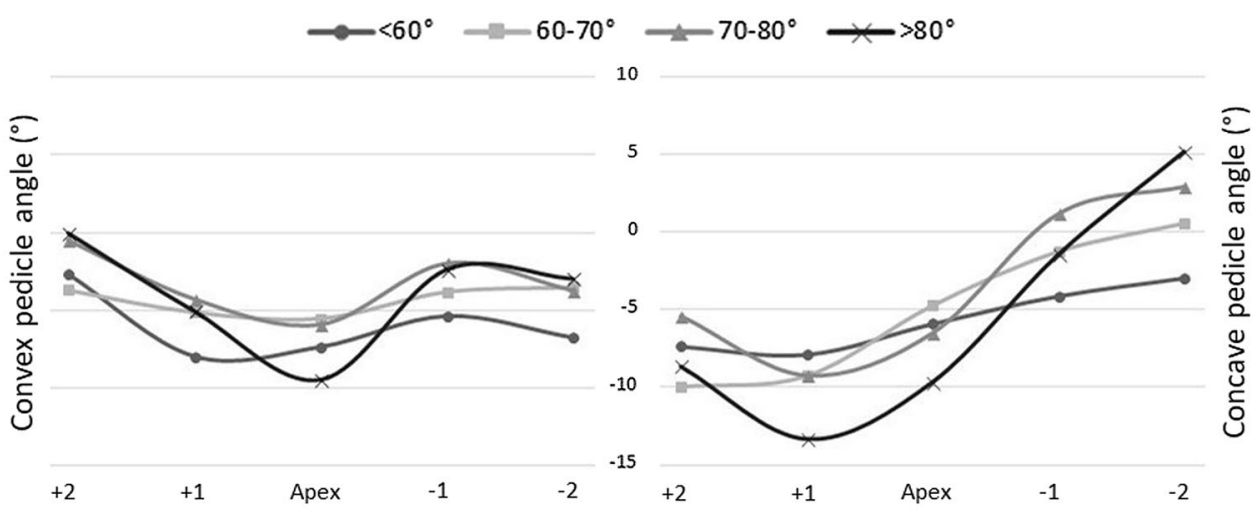

Sagittal pedicle-lamina angle

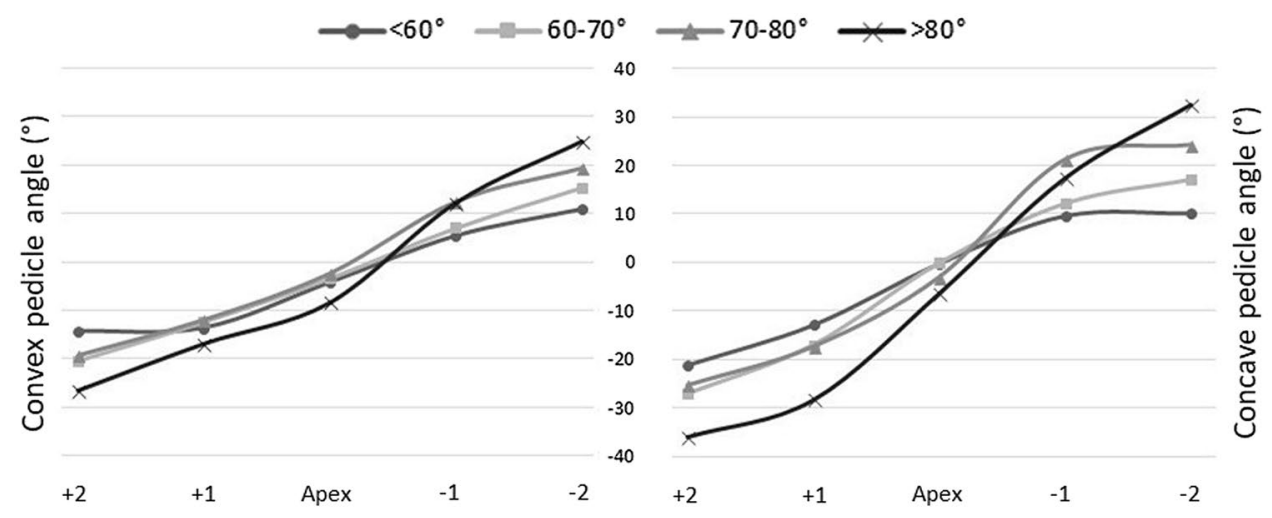

of this study is that the pads that are used during surgery are not used in the CT scanner, due to the size of the scanner. However, the angles, as given in this study, could serve as an approximate guideline for the expected direction of the pedicle screw. The ideal entry points, including the entry point shift due to the extra-pedicle trajectory, are part of our further ongoing study.

\section{Conclusion}

The 3D spatial reference of the pedicle axis relative to the operating table and the lamina, as described in this study is important for better understanding of spine surgeons. Pedicle angulation differs between concave and convex, the position as relative to the apex, as well as between curves with different severities. The transverse and sagittal pedicle angles, as relative to the operating table and laminae, is a useful reference for better understanding of the distorted 3D morphology and orientation of the pedicles and facilitate the intraoperative pedicle screw placement during posterior spinal surgery in AIS. The pedicle angle as relative to the lamina is the most relevant for intraoperative use. The angles, as given in this study, could serve as an approximate guideline for the expected direction of the pedicle screw.

Funding K2M unrestricted research grant; Alexandre Suerman MD/ PhD grant.

\section{Compliance with ethical standards}

Conflict of interest RCB (grants from Alexandre Suerman MD/PhD grant, outside the submitted work), JFH (none), SdeR (none), MvS (other from MRIguidance BV, outside the submitted work), TPCS (none), MAV (none), WCWC (none), BKWN (none), RMC (grants from K2M, outside the submitted work), JCYC (none).

Ethical approval For this type of study, formal consent is not required. It was reviewed and approved by the local Medical Ethical Committee.

Open Access This article is licensed under a Creative Commons Attribution 4.0 International License, which permits use, sharing, adaptation, distribution and reproduction in any medium or format, as long as you give appropriate credit to the original author(s) and the source, provide a link to the Creative Commons licence, and indicate if changes were made. The images or other third party material in this article are 
included in the article's Creative Commons licence, unless indicated otherwise in a credit line to the material. If material is not included in the article's Creative Commons licence and your intended use is not permitted by statutory regulation or exceeds the permitted use, you will need to obtain permission directly from the copyright holder. To view a copy of this licence, visit http://creativecommons.org/licenses/by/4.0/.

\section{References}

1. Cheng JC, Castelein RM, Chu WC et al (2015) Adolescent idiopathic scoliosis. Nat Rev Dis Primers 1:15030

2. Adams W (1882) Lateral curvature of the spine, external characters and morbid anatomy (lecture 4). Lectures on the pathology and treatment of lateral and other forms of curvature of the spine. Churchill, London, pp 69-93

3. Nicoladoni C (1904) Anatomie und mechanismus der skoliose. In: Kocher, von Mikulicz K (eds) Bibliotheca Medica. Verlag von erwin nagele, Stuttgart

4. Roaf R (1966) The basic anatomy of scoliosis. J Bone Joint Surg $\mathrm{Br}$ 48:786-792

5. Liljenqvist UR, Link TM, Halm HF (2000) Morphometric analysis of thoracic and lumbar vertebrae in idiopathic scoliosis. Spine 25:1247-1253

6. Schlösser TP, van Stralen M, Brink RC et al (2014) Three-dimensional characterization of torsion and asymmetry of the intervertebral discs versus vertebral bodies in adolescent idiopathic scoliosis. Spine 39:E1159-E1166

7. Brink RC, Schlösser TP, Colo D et al (2017) Asymmetry of the vertebral body and pedicles in the true transverse plane in adolescent idiopathic scoliosis: a CT-based study. Spine Deform 5:37-45

8. Rajwani T, Bhargava R, Moreau M et al (2002) MRI characteristics of the neurocentral synchondrosis. Pediatr Radiol 32:811-816

9. Takeshita K, Maruyama T, Chikuda H et al (2009) Diameter, length, and direction of pedicle screws for scoliotic spine: analysis by multiplanar reconstruction of computed tomography. Spine 34:798-803

10. Chu WC, Yeung HY, Chau WW et al (2006) Changes in vertebral neural arch morphometry and functional tethering of spinal cord in adolescent idiopathic scoliosis-study with multi-planar reformat magnetic resonance imaging. Stud Health Technol Inform 123:27-33

11. Watanabe K, Lenke LG, Matsumoto M et al (2010) A novel pedicle channel classification describing osseous anatomy: how many thoracic scoliotic pedicles have cancellous channels? Spine 35:1836-1842

12. Parent S, Labelle H, Skalli W et al (2004) Thoracic pedicle morphometry in vertebrae from scoliotic spines. Spine 29:239-248

13. Barr SJ, Schuette AM, Emans JB (1997) Lumbar pedicle screws versus hooks. Results in double major curves in adolescent idiopathic scoliosis. Spine 22:1369-1379

14. Suk SI, Lee CK, Kim WJ et al (1995) Segmental pedicle screw fixation in the treatment of thoracic idiopathic scoliosis. Spine 20:1399-1405

15. Suk SI, Lee SM, Chung ER (2005) Selective thoracic fusion with segmental pedicle screw fixation in the treatment of thoracic idiopathic scoliosis: more than 5-year follow-up. Spine 30:1602-1609

16. Boos N, Webb JK (1997) Pedicle screw fixation in spinal disorders: a European view. Eur Spine J 6:2-18

17. Halm H, Niemeyer T, Link $T$ et al (2000) Segmental pedicle screw instrumentation in idiopathic thoracolumbar and lumbar scoliosis. Eur Spine J 9:191-197
18. Liljenqvist UR, Halm HF, Link TM (1997) Pedicle screw instrumentation of the thoracic spine in idiopathic scoliosis. Spine 22:2239-2245

19. Abul-Kasim K, Ohlin A, Strombeck A et al (2010) Radiological and clinical outcome of screw placement in adolescent idiopathic scoliosis: evaluation with low-dose computed tomography. Eur Spine J 19:96-104

20. Di Silvestre M, Parisini P, Lolli F et al (2007) Complications of thoracic pedicle screws in scoliosis treatment. Spine 32:1655-1661

21. Upendra BN, Meena D, Chowdhury B et al (2008) Outcome-based classification for assessment of thoracic pedicular screw placement. Spine 33:384-390

22. Suk SI, Kim WJ, Lee SM et al (2001) Thoracic pedicle screw fixation in spinal deformities: are they really safe? Spine 26:2049-2057

23. Belmont PJ Jr, Klemme WR, Dhawan A et al (2001) In vivo accuracy of thoracic pedicle screws. Spine 26:2340-2346

24. Sarlak AY, Tosun B, Atmaca H et al (2009) Evaluation of thoracic pedicle screw placement in adolescent idiopathic scoliosis. Eur Spine J 18:1892-1897

25. Kim YJ, Lenke LG, Cho SK et al (2004) Comparative analysis of pedicle screw versus hook instrumentation in posterior spinal fusion of adolescent idiopathic scoliosis. Spine 29:2040-2048

26. Upendra B, Meena D, Kandwal P et al (2010) Pedicle morphometry in patients with adolescent idiopathic scoliosis. Indian J Orthop 44:169-176

27. Liljenqvist UR, Allkemper T, Hackenberg L et al (2002) Analysis of vertebral morphology in idiopathic scoliosis with use of magnetic resonance imaging and multiplanar reconstruction. J Bone Joint Surg Am 84:359-368

28. Cui G, Watanabe K, Hosogane N et al (2012) Morphologic evaluation of the thoracic vertebrae for safe free-hand pedicle screw placement in adolescent idiopathic scoliosis: a CT-based anatomical study. Surg Radiol Anat 34:209-216

29. Hong JY, Suh SW, Easwar TR et al (2013) Clinical anatomy of vertebrae in scoliosis: global analysis in four different diseases by multiplanar reconstructive computed tomography. Spine J 13:1510-1520

30. Kuraishi S, Takahashi J, Hirabayashi H et al (2013) Pedicle morphology using computed tomography-based navigation system in adolescent idiopathic scoliosis. J Spinal Disord Tech 26:22-28

31. Suk SI (2011) Pedicle screw instrumentation for adolescent idiopathic scoliosis: the insertion technique, the fusion levels and direct vertebral rotation. Clin Orthop Surg 3:89-100

32. Kim YJ, Lenke LG, Cheh $\mathrm{G}$ et al (2005) Evaluation of pedicle screw placement in the deformed spine using intraoperative plain radiographs: a comparison with computerized tomography. Spine 30:2084-2088

33. Chung KJ, Suh SW, Desai S et al (2008) Ideal entry point for the thoracic pedicle screw during the free hand technique. Int Orthop 32:657-662

34. Zindrick MR, Wiltse LL, Doornik A et al (1987) Analysis of the morphometric characteristics of the thoracic and lumbar pedicles. Spine 12:160-166

35. Lenke LG, Edwards CC 2nd, Bridwell KH (2003) The Lenke classification of adolescent idiopathic scoliosis: how it organizes curve patterns as a template to perform selective fusions of the spine. Spine 28:S199-S207

36. Schlösser TP, van Stralen M, Chu WC et al (2016) Anterior overgrowth in primary curves, compensatory curves and junctional segments in adolescent idiopathic scoliosis. PLoS One 11:e0160267

37. Kashimoto T, Yamamuro T, Hatakeyama K (1982) Anatomical and biomechanical factors in the curve pattern formation of idiopathic scoliosis. Acta Orthop Scand 53:361-368 
38. Davis CM, Grant CA, Pearcy MJ et al (2017) Is there asymmetry between the concave and convex pedicles in adolescent idiopathic scoliosis? A CT investigation. Clin Orthop Relat Res 475:884-893

39. Brooks HL, Azen SP, Gerberg E et al (1975) Scoliosis: a prospective epidemiological study. J Bone Joint Surg Am 57:968-972

40. Wessberg P, Danielson BI, Willen J (2006) Comparison of cobb angles in idiopathic scoliosis on standing radiographs and supine axially loaded MRI. Spine 31:3039-3044

41. Lee MC, Solomito M, Patel A (2013) Supine magnetic resonance imaging cobb measurements for idiopathic scoliosis are linearly related to measurements from standing plain radiographs. Spine 38:E656-E661
42. Shi B, Mao S, Wang Z et al (2015) How does the supine MRI correlate with standing $\mathrm{X}$-ray of different curve severity in adolescent idiopathic scoliosis? Spine 40:1206-1212

43. Brink R, Colo C, Schlösser T et al (2017) Upright, prone and supine spinal morphology and alignment in adolescent idiopathic scoliosis. Scoliosis Spinal Disord 12:6

Publisher's Note Springer Nature remains neutral with regard to jurisdictional claims in published maps and institutional affiliations. 